\title{
Memristive devices based on mass printed organic resistive switching layers
}

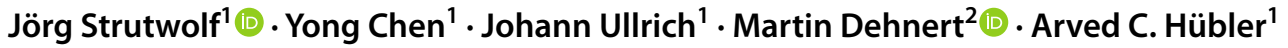

Received: 9 June 2021 / Accepted: 11 August 2021 / Published online: 25 August 2021

(C) The Author(s) 2021

\begin{abstract}
Resistive random-access memory is a candidate for next-generation non-volatile memory architectures. In this study, we use flexographic roll-to-roll printing technology for deposition of the resistive layer, a printing method that allows fast and cost-effective fabrication to create non-volatile resistive memory devices. Metal-free organic polymers blends composed of poly(methyl methacrylate) (PMMA) and a surplus of poly(vinyl alcohol) (PVA) serve as the active layer. Microscopic studies of the roll-to-roll printed layers show circular domains of PMMA embedded in PVA. The influence of the PMMA content in the polymer blend is investigated with respect to the performance and reliability of the resistive memory cells. Electrical characterization reveals a retention time of at least eleven days, a $R_{\text {off }} / R_{\text {on }}$ ratio of approx. two orders and write/ erase voltages of $+1 /-2 \mathrm{~V}$.
\end{abstract}

Keywords Resistive switching · Memristor · ReRAM - Organic polymer blend · Printed electronics · Flexible electronics · Roll-to-roll printing $\cdot$ Flexographic printing

\section{Introduction}

\subsection{State-of-the-art}

Over the last two decades printing processes have been established to fabricate low resolution flexible electronic devices $[1,2]$ based on printed active and passive electronic components such as diodes [3] and transistors [4, 5]. Fully printed multicomponent circuits have been realized [2], for example, ring oscillators [6,7], logic gates [8,9],

Jörg Strutwolf

joerg.strutwolf@mb.tu-chemnitz.de

Yong Chen

yong.chen@s2017.tu-chemnitz.de

Johann Ullrich

johann.ullrich@mb.tu-chemnitz.de

Martin Dehnert

martin.dehnert@physik.tu-chemnitz.de

Arved C. Hübler

arved.huebler@mb.tu-chemnitz.de

1 Institute for Print and Media Technology, Chemnitz University of Technology, 09107 Chemnitz, Germany

2 Institute of Physics, Chemnitz University of Technology, 09107 Chemnitz, Germany and amplifiers [10,11]. Printing as a manufacturing process has attributes, which are beneficial for memristive system fabrication. (i) The layout can be changed very easily so that different circuit layouts can be created and varied. (ii) By means of printing, these variants can be implemented quickly and cost-effectively in terms of production technology. (iii) The engineering possibilities in the framework of the printing process allow massive multi-layer systems, which also enable the realization of 3D circuits [12] in a memristive environment. (iv) The integration of other, nonmemristive components such as sensors, actuators, batteries is easily possible by printing. In addition, using foil substrates, printed memristive structures become important building blocks in emerging areas of flexible electronics such as soft electronic skin [13]. (v) Up-scaling in larger quantities is also conceivable. Therefore, over the last ten years, printing has been explored as an effective route towards memristive devices.

In fully printed memristive devices the bottom and top electrodes, as well as the memristive layer are printed. The development of fully printed memristive devices has been mainly focused on electrohydrodynamic (EHD) jet printing techniques for all three layers [14-17]. Partially printed memristive systems were fabricated on ITO coated polymer foil or glass which served as substrate and bottom electrode 
and memristive layers were deposited by EHD printing [18-22]. A combination of EHD printing for the memristive layer and EHD or screen printing for the top electrode and reverse offset printing for the bottom electrode was used by the Choi group [23-25]. The dominance of EHD printing methods for fabricating memristive structures is attributed to the high patterning resolution which can be achieved with this technique and which can extent below $100 \mathrm{~nm}$ [26]. Nevertheless, a number of fully printed memristive systems using the standard ink-jet technique [27-31] and aerosol jet printing $[32,33]$ have been reported.

\subsection{Our approach}

Here we report — to our knowledge for the first time—a resistive switching structure where the active layer is printed using a low-cost and time-efficient conventional printing method, namely flexo-graphic printing, a rotary in-line printing method for high-speed production typically used in the packaging industry $[34,35]$. It has been demonstrated that roll-to-roll printing technologies allow low cost and high volume production in printed electronics [36]. Due to the ability to print patterned films on flexible substrates at room temperature, flexography has been employed for fabrication in various areas of printed and flexible electronics, such as photovoltaics [37] and biosensors [38].

An obvious structure for memristors uses a PET foil coated with ITO (indium tin oxide) to serve as a bottom electrode. The circular-shaped top electrodes can be produced on the flexographically printed active layer by using a conventional printing process such as screen printing or flexographic printing. To simplify the experiments for the laboratory scale, a simple stencil printing technique was used here.

The active layer consists of a metal-free blend of the two organic polymers PVA (poly(vinyl alcohol)) and PMMA (poly(methyl methacrylate). Both polymers are inexpensive and nonhazardous, showing excellent transparency as well as bendability. PMMA and PVA have been used in memristive devices, mostly as organic compounds to realize organic-inorganic resistive switching layers. In such hybrid structures, PMMA or PVA serves as a matrix for embedding inorganic particles, for example, inorganic oxides such as $\mathrm{ZnO}$ nanoparticles and nanorods [39-43] $\mathrm{AlO}_{\mathrm{x}}$ and $\mathrm{HfO}_{\mathrm{x}}$ $[44,45], \mathrm{SnO}_{2}$ nanoparticles [46] as well as perovskites [22, 23, 47], 2-D material $\mathrm{MoS}_{2}$ [25], silver nanoparticles [48] and $\mathrm{Ag}$ ions [49]. The embedded inorganic particles form bulk-heterojunctions [50] and the wide electronic bandgap of the polymer matrix might restrict the movement of trapped charge carriers after disconnecting the electrical power and preserve the state of the memristive cell, leading to long retention times.
A few publications report on the use of PMMA only as active layers in memristive systems. No hysteresis and memory effect was found for the system ITO/PMMA/Al [48] and $\mathrm{Al} / \mathrm{PMMA} / \mathrm{Cu}$ [46]. Mangalam et al. [51] investigated the switching behaviour of PMMA with fluorine-doped tin oxide (FTO) glass substrates as the bottom electrode and Ag top electrodes. The authors observed resistive switching and identified $\mathrm{Ag}^{+}$filament formation through the PMMA as the origin of the switching process. They emphasized the importance of the annealing process for the solvent evaporation and subsequent vitrification of PMMA for an effective resistive switching with a two order increase in the current [51]. Resistive switching was also observed for a Pt/PMMA/ Ti structure with an $R_{\text {off }} / R_{\text {on }}$ ratio of ca. 10 [45]. In all cases, the PMMA layers were spin-coated.

In this work, we used a compressible mixture of PMMA and PVA without further particle doping as the active material between the two electrodes and investigated their memristive behaviour. The focus of this report is on the coating of the active material by a high-throughput printing technology.

\section{Materials and methods}

\subsection{Chemicals and instrumentation}

Poly(vinyl alcohol), PVA, (for synthesis, molecular weight $M_{\mathrm{w}} \approx 145 \mathrm{~kg} / \mathrm{mol}$, Merck), poly(methyl methacrylate), PMMA ( $M_{\mathrm{w}} \approx 15 \mathrm{~kg} / \mathrm{mol}, T_{\mathrm{g}}(\mathrm{DSC})=105^{\circ} \mathrm{C}$ (midpoint), Sigma-Aldrich) and dimethyl sulfoxide, DMSO (for HPLC, Sigma-Aldrich) were used as received. Indium tin oxide (ITO) coated PET foil (Melinex ST-504/Solutia ITO OC 50 $175 \mu \mathrm{m}$, sheet resistance $52 \Omega / \square$ ) served as substrate and inert bottom electrode (BE). Top electrodes (TE) were created from submicron particle silver ink (Loctite ECI 1011 E\&C, Henkel) and water-based poly(3,4-ethylenedioxythiophene) polystyrenesulfonate (PEDOT:PSS) ink (SV4, Heraeus Clevios $\mathrm{GmbH}$ ).

A Keithley 2612A sourcemeter (Tektronix) and a Süss PM5 probing station (Süss MicroTec) are utilized for electrical characterization. MATLAB ${ }^{\circ}$ scripts making use of the Instrument Control ToolboxTM (MathWorks) are employed to control the sourcemeter, for measurement automatization, data analysis, and visualization. For electrical measurements during mechanical (bending) stress a bending fixture that allows the variations of the curvature radius of the memristive devices was used, as described in [52]. Layer thicknesses are measured with a Dektak XT stylus profilometer (Bruker). Optical images are recorded with a Zeiss Axioskop 2 in transmission mode. Flexographic printing is carried out on a Flexiproof 100 (RK Printcoat Instruments). Atomic force microscopy (AFM) images were measured with a NanoWizard II® AFM (JPK Instruments). 


\subsection{Device fabrication}

To formulate ink for the resistive layer, blends of PMMA and PVA are dissolved in DMSO in a ratio of 1:9 (polymer blend:DMSO), followed by $30 \mathrm{~min}$ of shear mixing at room temperature. Before flexographic printing of the resistive layer, the ITO coated PET foil is rinsed with DI water, dried in a nitrogen stream, and sonicated for $10 \mathrm{~min}$ in ethanol. The polymer ink is transferred to the ITO-coated PET substrate by flexographic roll-to-roll printing under ambient conditions (room temperature $21{ }^{\circ} \mathrm{C}$ ), relative humidity of $(53 \pm 4) \%$. The principles of flexographic printing are illustrated in Fig. 1a. During printing, a fountain cylinder picks up the polymer blend and transfers it to an anilox cylinder. The surface of the anilox cylinder is engraved with micro-cavities, here with a cell volume of $39.1 \mathrm{~cm}^{3} /$ $\mathrm{m}^{2}$. The pyramidal micro-cavities ensure a uniform transport of a controlled amount of polymer blend to the plate cylinder. Excess of the polymer blend is removed from the anilox cylinder by a doctor blade. The desired pattern, in the present case areas of $15 \times 15 \mathrm{~mm}^{2}$, stands out as flexible reliefs on the plate cylinder's surface. The reliefs pick up the polymer blend from the anilox cylinder and printing occurs by pressing the reliefs to the substrate with the help of the impression cylinder. The printing speed is $30 \mathrm{~m} / \mathrm{min}$. Afterwards, the printed structures are annealed at $130{ }^{\circ} \mathrm{C}$ for five minutes. The PMMA:PVA blends were produced in a variation of $0,5,10,15$, and $20 \mathrm{wt} \%$ of PMMA. The different blends were used to fabricate various samples of the active layer. A higher percentage of PMMA fraction resulted in poor solubility and defects in the printed layers. The polymer blend layer thicknesses were estimated to be $(441 \pm 54) \mathrm{nm}$ (wt\% PMMA), $(541 \pm 114) \mathrm{nm}(10 \%)$, $341 \pm 56 \mathrm{~nm}(15 \%)$, and $(314 \pm 86) \mathrm{nm}(20 \%)$ from stylus profilometer measurements by measuring six printed samples of each polymer blend at three different locations, see Figure S1 of the Supplemental Information. Several factors for a given ink lead to variations in the thickness of roll-toroll printed layers. Ambient conditions (temperature, humidity) were kept constant or within limits during the printing process. However, irregularities in the layer thickness are also caused by non-fully automated ink feeding and variation of the printing pressure, as was observed recently [53]. A reproducible layer thickness control within a few $\mathrm{nm}$ is difficult to accomplish in roll-to-roll printing technology and is a disadvantage compared to clean room techniques (e. g. vapour deposition, ALD), but also non-contact printing techniques, like electrohydrodynamic printing. On the other hand, thicknesses of 300-500 $\mathrm{nm}$ are relatively large for memristive layers and irregularities might have less influence on memristive properties compared to layers with thickness $<50 \mathrm{~nm}$. The top electrodes are fabricated by stencil printing. PET foils with a square pattern of $3 \times 3$ circular holes are used as stencils. Placing the stencils on top of the resistive layer and spreading the silver ink over the stencils using a squeegee creates the top electrodes in a $3 \times 3$ pattern. The electrode diameter is $(0.83 \pm 0.07) \mathrm{mm}$, and the centre-to-centre electrode distance is $3 \mathrm{~mm}$. The device is placed again in an oven $\left(5 \mathrm{~min}\right.$ at $\left.130{ }^{\circ} \mathrm{C}\right)$ for annealing the submicron particle silver ink. Figure $1 \mathrm{~b}$ shows a diagram of the memristive device. Alternatively PEDOT:PSS is used for top electrodes and deposited following the same method as silver top electrodes. After deposition, PEDOT:PSS is annealed at $130{ }^{\circ} \mathrm{C}$ for five minutes.
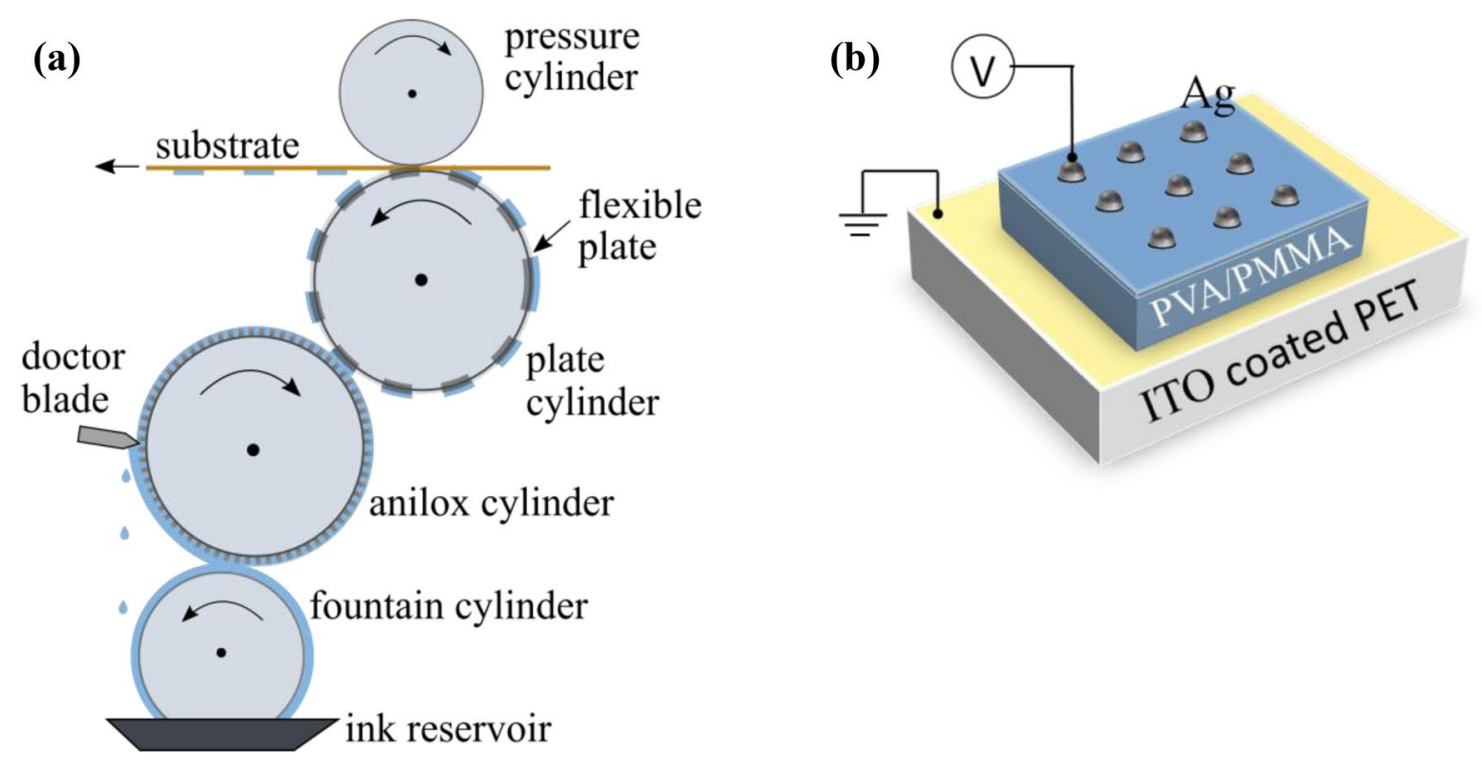

Fig. 1 a Illustration of the flexographic printing process; $\mathbf{b}$ diagram of the memristive device 


\section{Results and discussion}

\subsection{Structural and optical characterization of the memristive layer}

Before focusing on the electrical characterization, we investigate the morphology of the printed polymer blend by optical microscopy and AFM. Compatibility of PMMA was found previously for PMMA:PVA mixtures if the PVA content was higher than $60 \mathrm{wt} \%$ [54]. Compatibility denotes the forming of thermodynamically miscible phases. However, in [54] the polymer blend was heated to a maximum of $50{ }^{\circ} \mathrm{C}$, which is well below the midpoint
Fig. 2 Optical microscopic images of PMMA:PVA blend surfaces. PMMA content (wt\%): a 0 , b 5, c 10 , d 15 and e 20 .

The scale bar in a applies to all five images

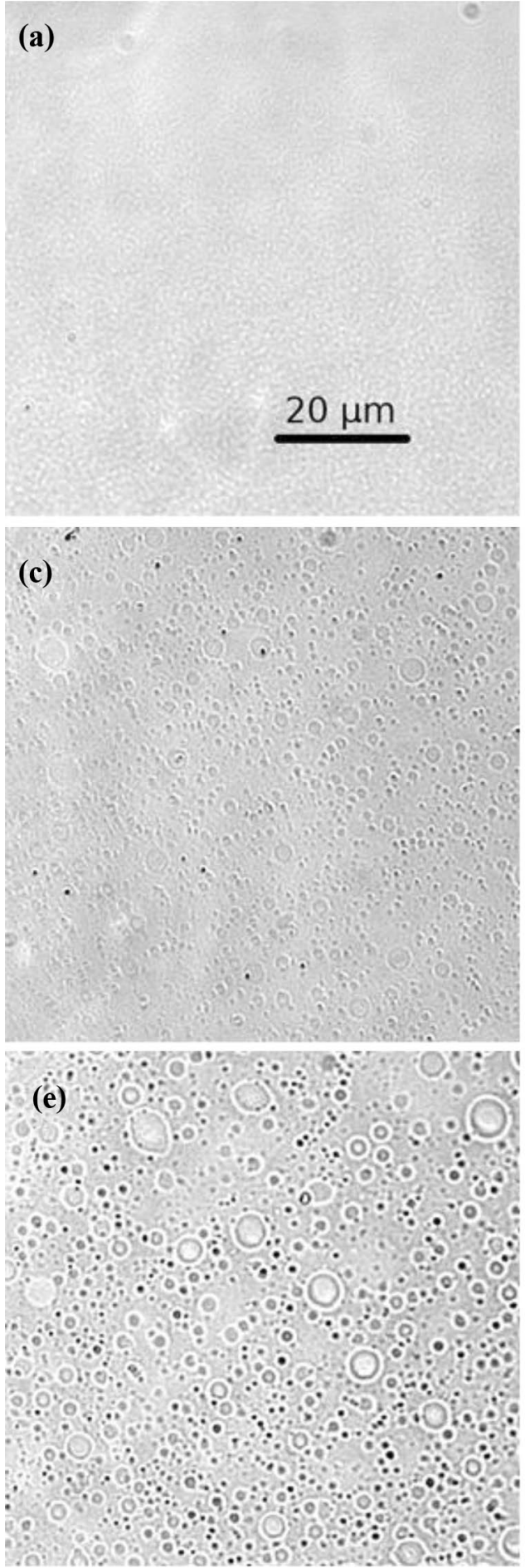

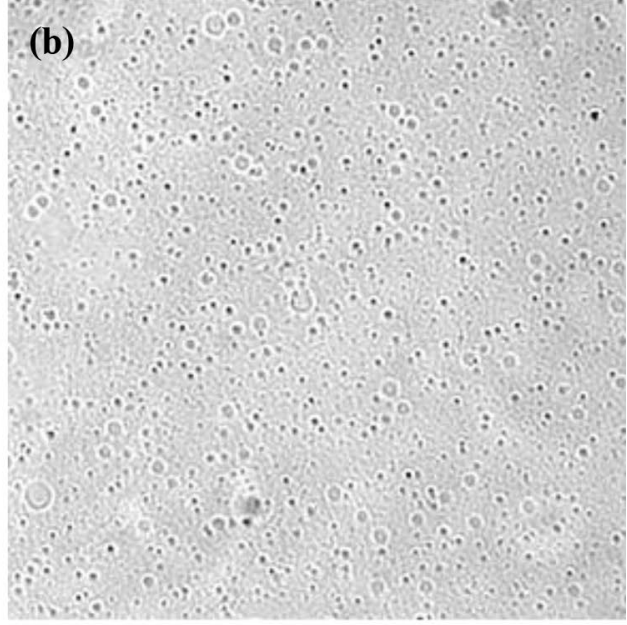

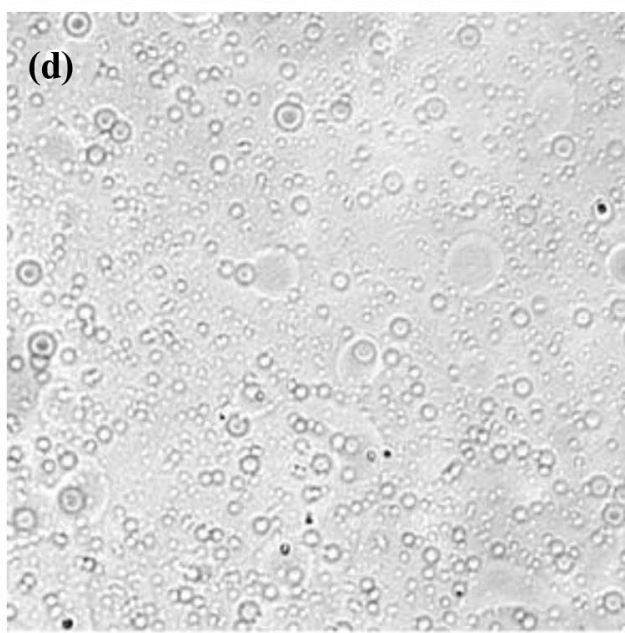


glass transition temperature, $T_{\mathrm{g}}$, of PMMA. Figure 2 presents optical images of flexographically printed and annealed polymer blend samples with a PMMA content of $0,5,10,15$, and $20 \mathrm{wt} \%$. We observe uniformly distributed circular domains, likely to be PMMA, even at the lowest PMMA content of $5 \mathrm{wt} \%$. The size of the domains increases with increasing PMMA concentration. The PVA background shows an inhomogeneous structure in the form of brightness fluctuations, which is due to the visible effect of viscous fingering. The phenomena of viscous fingering due to ink splitting between the printing cylinder and substrate are well documented for flexographic printing $[55,56]$, and the finger-like features along the print have been attributed to the Taylor-Saffman instability [57]. In a separate test, the polymer blend was deposited using drop coating to rule out the possibility that the circular domains seen in Fig. 2 are due to the flexographic printing process. However, the microscopic images of drop-coated polymer blend layers show the same features as the flexographic printed layers (see Figure S2 in the Supplemental Information).

Amplitude modulation (AM) AFM imaging was performed to investigate the morphology and the phase separation on a smaller length scale than optical microscopy. Figure 3 shows AM-AFM height images with dimensions of $50 \times 50 \mu \mathrm{m}^{2}$ with a PMMA content of 5, 10, 15, and $20 \mathrm{wt} \%$.
The height images are a 3D-rendered heightfield with the height as texture and illuminated from the right-hand side to enhance small surface corrugation with shadows. PMMArich domains are more specular than PVA-rich domains. The circular domains observed in Fig. 2 are also visible despite smaller circular domains in the range of $\approx 700 \mathrm{~nm}$ in diameter for $5 \mathrm{wt} \%$ PMMA and $\approx 900 \mathrm{~nm}$ for $20 \mathrm{wt} \%$ PMMA. We attribute these circular domains to PMMA-rich domains. They are visible as smooth domains in the AMAFM height image and they show a different phase contrast in the AM-AFM phase image (see Figure S3 in the Supplemental Information).

The root mean square (RMS) roughness increases with increasing PMMA content (Fig. 3e). Between 10 and $20 \mathrm{wt} \%$ PMMA, the RMS roughness is nearly constant. Figure $3 \mathrm{f}$ shows the PMMA surface coverage as a function of the PMMA content. The PMMA surface coverage is calculated as the cumulative area of the PMMA domains identified in the AM-AFM phase image divided by the area of the AMAFM image. Remarkably, it is always larger than the PMMA content in the blend itself.

With this, we conclude that PMMA forms small PMMArich domains floating on the surface on PVA-rich domains. Large PMMA-rich domains connect the bottom electrode with the top electrode. The increased roughness indicates an increased phase separation between PVA-rich domains
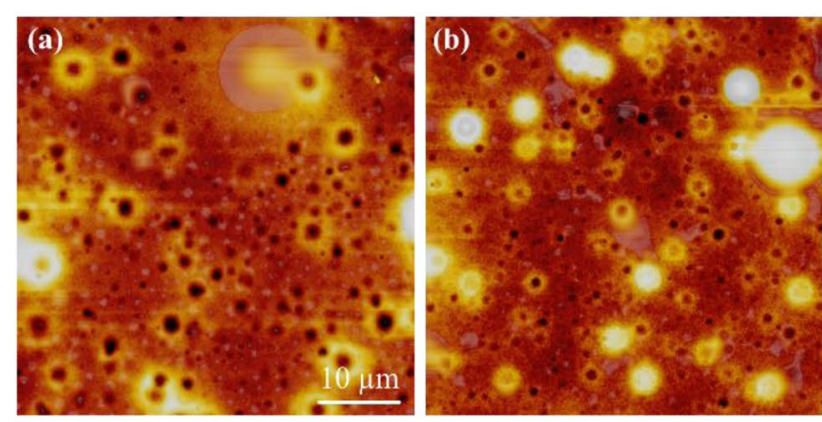

(e)

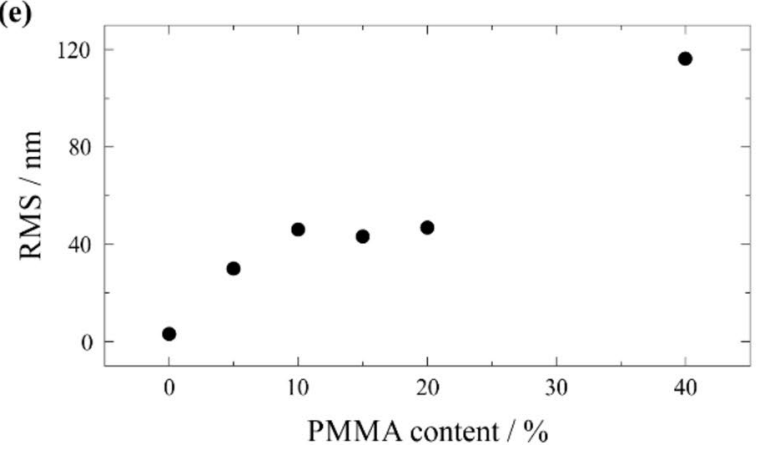

Fig. 3 AM-AFM height image of PMMA:PVA blend surfaces. PMMA content (wt\%): a 5, b 10, c 15 and d 20. The height images are a 3D-rendered heightfield with the height as texture and illuminated from the right-hand side to enhance small surface corrugation with shadows. PMMA-rich domains are more specular than PVA-rich
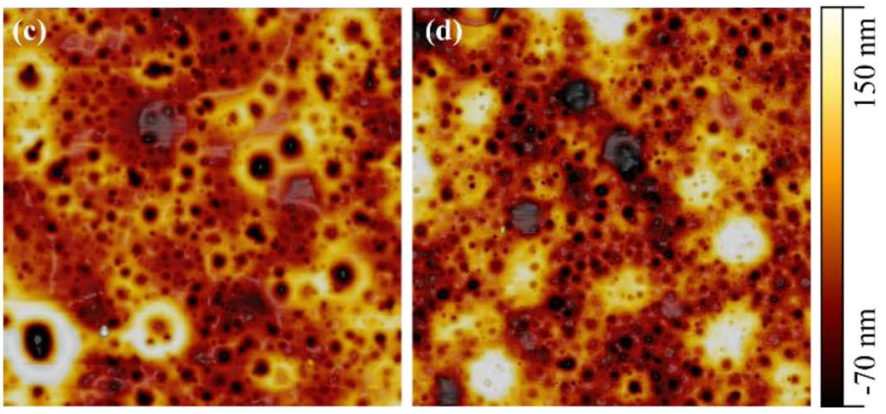

(f)

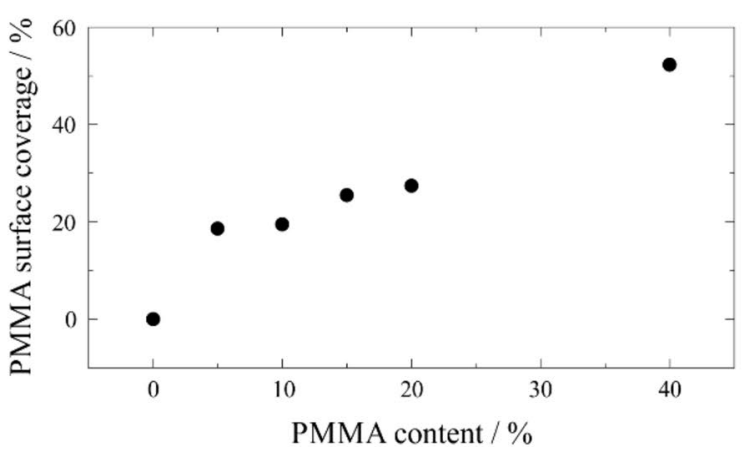

domains. The scale bar in a applies to all four images. e Root mean square roughness of all AM-AFM height images as a function of the PMMA content. $\mathbf{f}$ Relative PMMA surface coverage as a function of the PMMA content extracted from AM-AFM images 
and PMMA-rich domains with larger PMMA content [58]. Also, the size of the PMMA-rich domains increases with increasing PMMA content.

To support the conclusion that larger PMMA-rich domains connect the bottom and top electrode, we remove a part of the active layer from the PET foil (see Figure S4 in the Supplemental Information). We used a needle to scratch the PMMA:PVA layer. As a result, the layer is folded back (yellow area in Figure S4b), leaving droplets of the PMMArich domains on the PET foil (blue area in Figure S4b). The height of these droplets $(\approx 400 \mathrm{~nm})$ is in the range of the film thickness $(\approx 500 \mathrm{~nm})$. We suggest not all PMMA-rich droplets adhere to the PET foil. More PMMA-rich domains are visible in the intact area of the active layer (orange area in Figure $\mathrm{S} 4 \mathrm{~b}$ ).

\subsection{Electrical performance}

The resistive switching behaviour of the ITO/PMMA:PVA blend/Ag based memory is investigated by DC triangular voltage scans. During the measurements, the bias voltage was applied to the Ag top electrode, while the ITO bottom electrode was grounded. The voltage was swept in the sequence $0 \rightarrow$ positive $\rightarrow 0 \rightarrow$ negative $\rightarrow 0$, with a compliance current of $2.5 \mathrm{~mA}$ to protect the device from a hard breakdown. Figure 4a displays semi-logarithmic $I-V$ characteristic curves, showing bi-polar switching behaviour. At the start of the scan $(0 \mathrm{~V})$ the device is in a high resistive state (HRS) also referred to as the OFF state, switching to a low resistive state (LRS) referred to as the ON state at a positive voltage $V_{\text {set }}$. During the negative-going scan, the device remains in the LRS before a reset into the HRS occurs at a negative voltage. Starting with a pristine device, the first voltage scan (red line in Fig. 4a) requires a higher voltage value for switching into the LRS ( $V_{\text {set }} \approx 1.2 \mathrm{~V}$ ) compared to subsequently following scans due to the initial forming process of the conducting path. After the forming step, a lower $V_{\text {set }}$ value of around $1 \mathrm{~V}$ is observed, revealing a low writing voltage of the memory device. Only for the forming cycle, the compliance current is limiting the current flow through the device, while for the following voltage cycles the maximum measured current of approx. $1 \mathrm{~mA}$ is below the pre-set compliance current. If the compliance voltage was set too low (less than $0.25 \mathrm{~mA}$ ), the $\mathrm{ON}$ state was not stable, resulting in volatile memory behaviour. This is contributed to the formation of unstable conducting filaments due to the limited growth of the filaments [59]. As shown in Fig. $4 \mathrm{a}$ the reset voltage, $V_{\text {reset }}$, is not well defined, instead, the reset appears gradually with the reset to HRS clearly put into effect at $-2 \mathrm{~V}$. The active layers with 5 and 10 PMMA wt $\%$ content show poor endurance performance with only $15 \%$ (for $5 \mathrm{wt} \%$ PMMA) and 33\% (for $10 \mathrm{wt} \%$ PMMA) from 100 consecutive voltage cycles resulting in a clear set/reset characteristics, while the performance of $15 \%$ PMMA and 20\% PMMA is much better (> 80\%). The basic features of the $I-V$ curves for PMMA-PVA blends of 10, 15, and $20 \mathrm{wt} \%$ are almost identical, as shown in Figure S5 of the Supplemental Information. The cumulative probability of the set voltages for the polymer blends is presented in Fig. 4b. $V_{\text {set }}$ shifts slightly with increasing PMMA content to higher values, except for the 5\% PMMA blend, which also shows the biggest spreading of the $V_{\text {set }}$ values. This goes along with poorer switching reliability for the layer with the lowest PMMA content of 5\% but also $10 \%$, as will be shown later. The setting voltages for the $20 \mathrm{wt} \%$ PMMA layer spread between 0.8 and $1.1 \mathrm{~V}$. The variability of $V_{\text {set }}$ is commonly associated with the stochastic nature of metallic filament formation and has been observed in organic-based resistive random-access memory (RRAM) $[60,61]$. In the case of the absence of PMMA, no hysteresis and resistive switching are observed, in agreement with a previous report for the system Ag/PVA/ITO [62]. The insulating PVA acts as a charge blocking medium in the composite. For blends of $40 \mathrm{wt} \%$ PMMA, Fig. 3e shows a strong increase in the surface roughness due to the poor solubility of PMMA in the polymer blend ink. Printed layers with PMMA content larger than $40 \mathrm{wt} \%$ show defects. However, the RMS roughness of 10, 15, and 20\% PMMA has similar values. Since PMMA is the active component for filament formation, one would expect a decrease in the setting voltage with increasing PMMA content. For memristive layers of pure PMMA ( $240 \mathrm{~nm}$ thick) with active electrodes of Ag switching voltage of $\approx 2 \mathrm{~V}$ were reported, which is about twice the value measured for the PMMA/PVA blends though the layer thickness was smaller. The lower $V_{\text {set }}$ values shown in Fig. $4 \mathrm{~b}$ might be a hint that the filament formation along the PMMA/PVA phase boundaries plays an important role in the switching ON process, and a high PMMA content does not necessarily imply a lower set voltage. Furthermore, it has been shown that for metallic filament formation in organic layers since the ion conductivity is substantially lower and the polymer thickness is much thicker, the drift of metalcations is expected to be much slower than that in inorganic metallization cells [63]. Hence, the migration of metal-cations rather than the redox reaction predominantly governs the conduction filament growth. Thus, organic layers with larger free volumes exhibit lower operation voltages [64]. The interface between PVA and PMMA can supply nanosized voids and cavities, which provide preferential paths for conducting filament formation.

The cumulative probabilities of the resistant values of the HRS $\left(R_{\text {off }}\right)$ and LRS $\left(R_{\text {on }}\right)$ for organic blends containing 10 , 15 , and $20 \mathrm{wt} \%$ PMMA are displayed in Fig. $4 \mathrm{c}$ The $R_{\text {off }} / R_{\text {on }}$ ratio for the 10\% PMMA blend is in average smaller than for the other two blends. Furthermore, it can be observed that the 20\% PMMA sample shows more uniform resistance in 

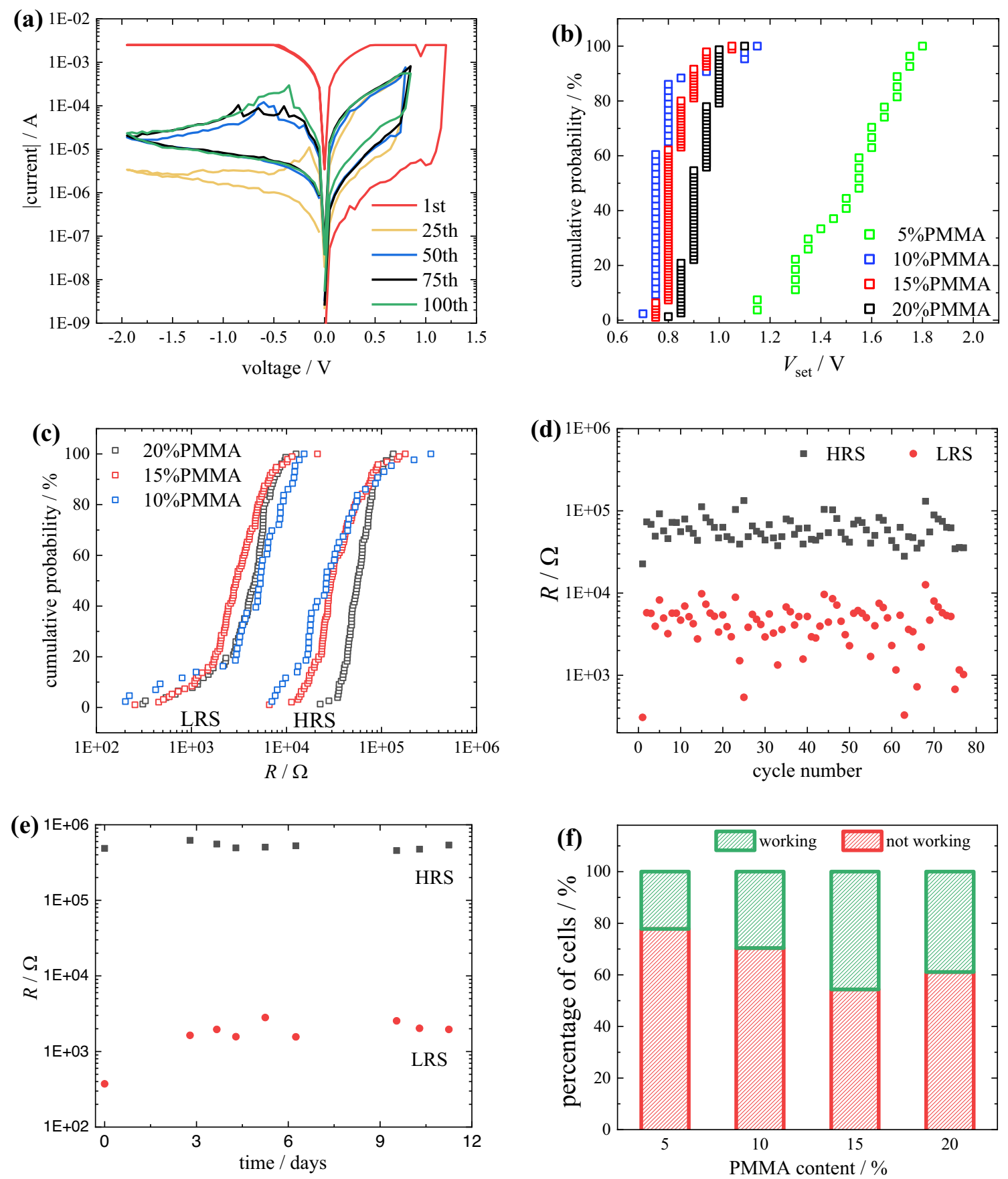

Fig. 4 a The 1st, 25th, 50th, 75th and 100th $I-V$ curve of 100 continuous resistive switching cycles for the ITO/PMMA:PVA/Ag device (20\% PMMA). b The cumulative probabilities of the set voltages for active layers with PMMA contents as indicated. c Cumulative probabilities of the resistance of the ON (LRS) and OFF (HRS) states. d

HRS and LRS compared to the other two samples. Figure 4d shows the first 80 endurance cycles for a $20 \mathrm{wt} \%$ PMMA active layer. The resistive states were estimated at a reading bias of $100 \mathrm{mV}$. Despite the variations of the resistance in
On and OFF resistances of the first 80 switching cycles. The reading voltage was $100 \mathrm{mV}$. e Retention time measurement for a $20 \%$ PMMA device. f Switching reliability taking into account crossdevice and cross-cell measurements

particular for the LRS, clear discrimination between the LRS and HRS is possible.

Voltage pulse controlled retention time measurements were performed, and Fig. 4e presents an example for an 11-day test run using a polymer blend with 20\% PMMA. 
Initially, the memristive cell was set in an LRS or HRS by applying a voltage pulse of $+2 \mathrm{~V}$ or $-2 \mathrm{~V}$. The resistive state was then monitored for 11 days by applying reading pulses of $200 \mathrm{mV}$. The pulse width in each case was $0.01 \mathrm{~s}$. The resistance of the HRS state remains at an almost constant level, while the resistance of the LRS shows some nonsystematic variability over the period of 11 days. Starting with a $R_{\text {off }} / R_{\text {on }}$ ratio of $\approx 3$ orders, the ratio decreases and remains at $\approx 2.5$ orders. The reason for the decay might be the highly mobile nature of the $\mathrm{Ag}$ cations with a relatively low diffusion barrier leading to long-term instabilities of the conducting filaments. This issue becomes more relevant since the active layer with an average thickness of $400 \mathrm{~nm}$ is relatively thick and longer filaments have to be formed to maintain the LRS. Still, a $R_{\text {off }} / R_{\text {on }}$ ratio between 2 and 3 orders might be sufficient for memory application.

The cross-device and cross-cell switching cycle reliability were evaluated for devices with PMMA:PVA blends containing 5, 10, 15 and $20 \mathrm{wt} \%$ PMMA. For each blend formulation, six devices were fabricated and for each device, all nine cells were evaluated, in total $6 \times 9$ cells for each polymer. The testing procedure consists of measuring the first five switching cycles. Working in Fig. $4 \mathrm{f}$ indicates that five of five cycles in each measurement of the $6 \times 9$ cells showed proper set and reset behaviour, i. e. an $I-V$ curve with pinched hysteresis, clear discrimination between HRS and LRS, and bipolar switching behaviour. If one or more cycles failed, the cell is classified as not working. Failure is due to voltage cycles showing no switching off (reset) process at negative voltages after the set process, resulting in a permanent electrical contact of the top and bottom electrode (short circuit), though in many cases the reset process occurs again at the following cycle bringing the device back to working condition. However, such devices are also qualified as not working. Not working also applies for cells showing short circuits from the beginning on, due to printing failures. For the $5 \mathrm{wt} \%$ PMMA blend $21 \%$ of the cells showed proper switching behaviour for all voltage cycles. These percentages increase to $45 \%$ and $40 \%$ for $15 \mathrm{wt} \%$ and $20 \mathrm{wt} \%$ PMMA blends, respectively. Taking into account that the test considers cross-cell as well as cross-device reliability, a success rate of $40 \%$ seems satisfactory, but leaves room for improvement, e.g. by performing the flexographic printing process in a better-controlled environment, since temperature and humidity influenced the consistency of printing quality.

Figure 5 presents the performance of the resistive switching device under bending stress. Figure S6 of the Supplemental Information illustrates the bending fixture according to [52]. The bending radius $r$ is approximated by $r=b^{2} / 8 / h$ [52] and was changed from 160 to $30 \mathrm{~mm}$. The bending radius down to $30 \mathrm{~mm}$ has no significant and systematic influence on the ON and OFF resistive states of the memory

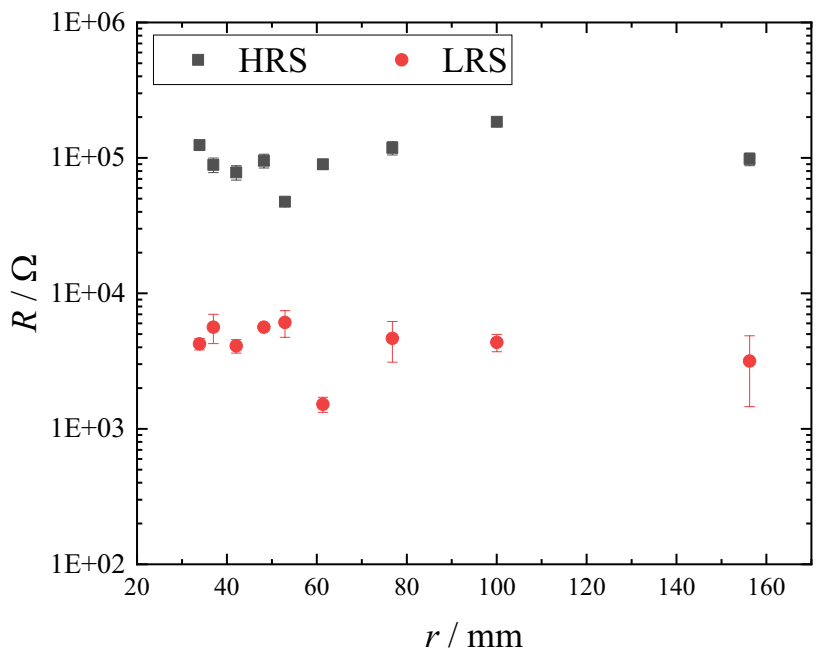

Fig. 5 High and low resistance states of a memristive cell during bending. The $R_{\text {on }}$ and $R_{\text {off }}$ values are the mean and the corresponding SD of five consecutive switching cycles at each bending radius $r$

cell. This is attributed to the flexibility of the organic polymer blend-based active layer and the embedded Ag nanowires as well as to the high thickness of approx. $400 \mathrm{~nm}$ of the active layer, which possibly prevents short-circuits and permanent damage.

Silver as an active electrode material and ITO as an inert electrode suggests that the formation and dissolution of metallic filaments play a major role in the observed resistive switching of the Ag/PMMA:PVA/ITO device. Devices were fabricated where the conducting polymer poly(3,4-ethylenedioxythiophene) polystyrenesulfonate (PEDOT:PSS) is replacing $\mathrm{Ag}$ as the material for the top electrode. PEDOT:PSS serves as an inert electrode, in contrast to the active silver electrode. The recorded $I-V$ curves showed no pinched hysteresis, Fig. 6a. It is reasonable to assign the device to the class of electrochemical metallization memory cells [63]. To gain some more insight into the conduction mechanism of the organic blend device, the positive voltage region of a typical $I-V$ curve (in this case the 5 th cycle after the forming process in a continuous cycling experiment) is plotted in a double logarithmic scale, as shown in Fig. 6 b. The $I-V$ curves follow a general power-law behaviour $I \propto V^{\mathrm{m}}$. At low bias in HRS and LRS slopes of $m \approx 1$ are observed, that is the current is linearly proportional to the voltage $(I \propto V)$ and the current is dominated by ohmic conduction. The ohmic behaviour of the ON state (LRS) suggests the conducting filament formation during the set process, while the ohmic conductance at low bias in the HRS might be caused by the residual conductive filaments after the reset process [65]. At $V_{\mathrm{tr}} \approx 0.4 \mathrm{~V}$ the slope in the HRS changes to $m \approx 2$, indicating the transition to a space charge limited current (SCLC) according to Child's square 


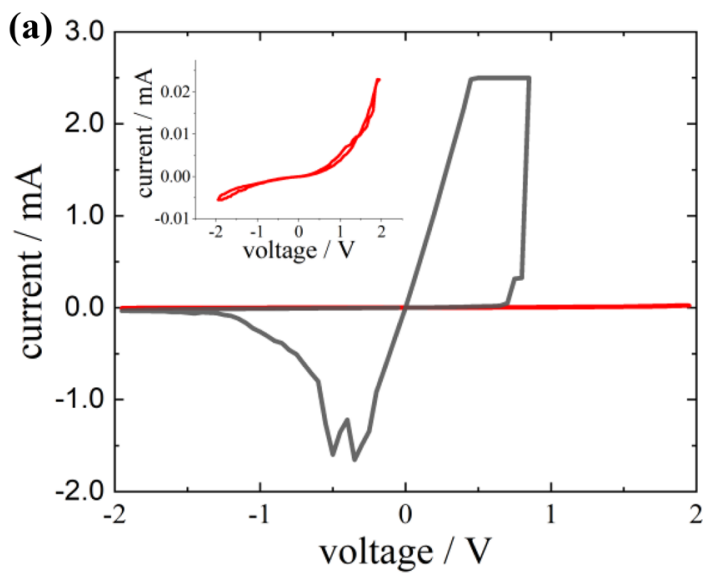

Fig. 6 a $I-V$ measurement for $20 \mathrm{wt} \%$ PMMA active layer. The materials of the TE are Ag (black line) and PEDOT:PSS (red line); the inset shows the same $I-V$ curve for the PEDOT:PSS TE on a smaller

law. The $V_{\text {tr }}$ value is in agreement with the value measured by Mangaram et al. [51] for the system Ag/PMMA/FTO. At $\approx 1 \mathrm{~V}$ the current steeply increases up to the limit set by the compliance current, suggesting that during the set process a conductive path is created within PMMA and along the boundaries between the PMMA domains and PVA. For the spin-coated PMMA film reported in [51], a further transition to ohmic conduction is observed at $V_{\mathrm{tr}}=1.4 \mathrm{~V}$ followed by switching to the LRS at $V_{\text {set }} \approx 2 \mathrm{~V}$. This transition is not observed in the present PMMA:PVA device because $V_{\text {set }}$ occurs already at $1 \mathrm{~V}$. Note that the slope $m$ of the LRS in the positive bias region during the voltage-decreasing sweep does not abruptly drop to $m \approx 1$ after the compliance current limitation, but approach $m \approx 1$ at about $0.4 \mathrm{~V}$ with a steeper slope, indicating that SCLC is relevant before the onset of ohmic conduction in the LRS. This behaviour seems to be particular for PMMA as it is also seen in Fig. 3a of reference [51]. Further investigation will be needed to clarify.

\section{Conclusions}

The flexographic roll-to-roll printing technique has been successfully applied to create a memristive layer based on metal-free low-cost polymer blends. The device shows decent $R_{\text {off }} / R_{\text {on }}$ values and good retention times. The structural characterization of the PMMA:PVA blend suggests that the top and bottom electrodes are connected via continuous domains of PMMA embedded in PVA. These serve as pathways for metallic filament formation and dissolution which dominates the switching behaviour.

By its very nature, printing technology is designated for high throughput multilayer deposition. The conceptual study presented here was focused on the application

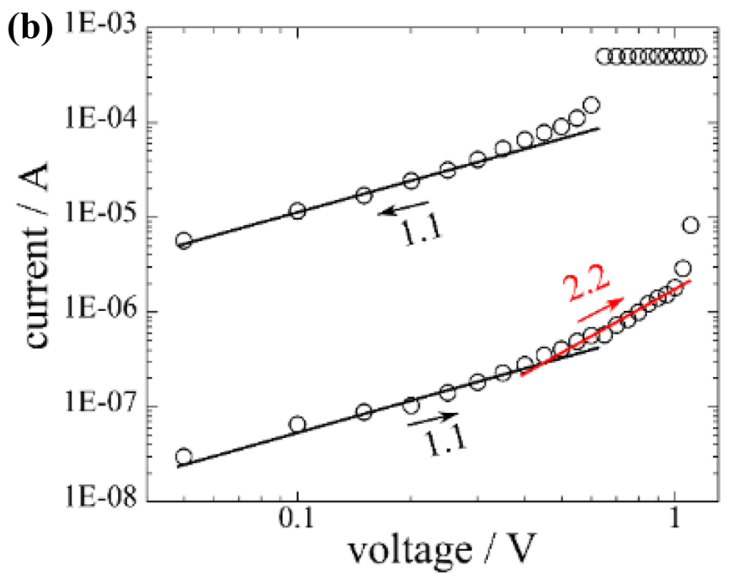

current scale. b $\log (I)-\log (V)$ plot with numbers indicate the slopes of the fitted straight lines

of a roll-to-roll printing technique for depositing the active layer. The bottom electrode was not printed, instead, an ITO coated foil was employed. Despite the convenience and widespread use, ITO has the disadvantage of relatively high price which is expected to increase further due to the low abundance and high cost of extraction of indium [66]. In future work, we aim to develop a fully mass-printed flexible memristive device for applications that require mass production at a low cost and for which the storage density is not crucial. An example of such an application is a rewritable non-volatile memory for encrypted authentication solutions of RFID tags.

Supplementary Information The online version contains supplementary material available at https://doi.org/10.1007/s00339-021-04851-9.

Funding Open Access funding enabled and organized by Projekt DEAL. This work was funded by the European Union, by the European Regional Development Fund (ERDF) and by the Free State of Saxony.

\section{Declarations}

Conflict of interest The authors declare that they have no conflict of interest or competing interests.

Data availability Data are available from the corresponding author upon request.

Open Access This article is licensed under a Creative Commons Attribution 4.0 International License, which permits use, sharing, adaptation, distribution and reproduction in any medium or format, as long as you give appropriate credit to the original author(s) and the source, provide a link to the Creative Commons licence, and indicate if changes were made. The images or other third party material in this article are included in the article's Creative Commons licence, unless indicated otherwise in a credit line to the material. If material is not included in the article's Creative Commons licence and your intended use is not 
permitted by statutory regulation or exceeds the permitted use, you will need to obtain permission directly from the copyright holder. To view a copy of this licence, visit http://creativecommons.org/licenses/by/4.0/.

\section{References}

1. R.A. Street, T.N. Ng, D.E. Schwartz, G.L. Whiting, J.P. Lu, R.D. Bringans, J. Veres, From printed transistors to printed smart systems. Proc. IEEE 103, 607-618 (2015). https://doi.org/10.1109/ JPROC.2015.2408552

2. J.S. Chang, A.F. Facchetti, R. Reuss, A circuits and systems perspective of organic/printed electronics: review, challenges, and contemporary and emerging design approaches. IEEE J. Emerg. Sel. Top Circuits Syst. 7, 7-26 (2017). https://doi.org/10.1109/ JETCAS.2017.2673863

3. Y. Chu, C. Qian, P. Chahal, C. Cao, Printed diodes: materials processing, fabrication, and applications. Adv. Sci. 6, 1801653 (2019). https://doi.org/10.1002/advs.201801653

4. B. Kang, W.H. Lee, K. Cho, Recent advances in organic transistor printing processes. ACS Appl. Mater. Interfaces 5, 2302-2315 (2013). https://doi.org/10.1021/am302796z

5. W. Tang, Y. Huang, L. Han, R. Liu, Y. Su, X. Guo, F. Yan, Recent progress in printable organic field effect transistors. J. Mater. Chem. C 7, 790-808 (2019). https://doi.org/10.1039/C8TC0 $5485 \mathrm{~A}$

6. H. Kempa, M. Hambsch, K. Reuter, M. Stanel, G.C. Schmidt, B. Meier, A.C. Hübler, Complementary ring oscillator exclusively prepared by means of gravure and flexographic printing. IEEE Trans. Electron Devices. 58, 2765-2769 (2011). https://doi.org/ 10.1109/TED.2011.2153856

7. J. Zhou, T. Ge, E. Ng, J.S. Chang, Fully additive low-cost printed electronics with very low process variations. IEEE Trans. Electron Devices 63, 793-799 (2016). https://doi.org/10.1109/TED.2015. 2508484

8. M. Hambsch, K. Reuter, H. Kempa, A.C. Hübler, Comparison of fully printed unipolar and complementary organic logic gates. Org. Electron 13, 1989-1995 (2012). https://doi.org/10.1016/j. orgel.2012.05.045

9. P.C. Hütter, T. Rothländer, G. Scheipl, B. Stadlober, All screenprinted logic gates based on organic electrochemical transistors. IEEE Trans. Electron Devices 62, 4231-4236 (2015). https://doi. org/10.1109/TED.2015.2491342

10. J. Chang, X. Zhang, T. Ge, J. Zhou, Fully printed electronics on flexible substrates: High gain amplifiers and DAC. Org. Electron. 15, 701-710 (2014). https://doi.org/10.1016/j.orgel.2013.12.027

11. B. Kheradmand-Boroujeni, G.C. Schmidt, D. Höft, M. Bellmann, K. Haase, K. Ishida, R. Shabanpour, T. Meister, C. Carta, P. Ghesquiere, A.C. Hübler, F. Ellinger, A fully-printed self-biased polymeric audio amplifier for driving fully-printed piezoelectric loudspeakers. IEEE Trans. Circuits Syst. I Regul. Pap. 63, 785-794 (2016). https://doi.org/10.1109/TCSI.2016.2538060

12. A.C. Hübler, G.C. Schmidt, H. Kempa, K. Reuter, M. Hambsch, M. Bellmann, Three-dimensional integrated circuit using printed electronics. Org. Electron. 12, 419-423 (2011). https://doi.org/10. 1016/j.orgel.2010.12.010

13. M. Soni, R. Dahiya, Soft eSkin: distributed touch sensing with harmonized energy and computing. Philos. Trans. R Soc. A Math. Phys. Eng. Sci. 378, 20190156 (2020). https://doi.org/10.1098/ rsta.2019.0156

14. N. Duraisamy, N.M. Muhammad, H.C. Kim, J.D. Jo, K.H Choi, Fabrication of TiO 2 thin film memristor device using electrohydrodynamic inkjet printing. Thin Solid Films 520, 5070-5074 (2012). https://doi.org/10.1016/j.tsf.2012.03.003

15. S. Ali, J. Bae, C.H. Lee, K.H. Choi, Y.H. Doh, All-printed and highly stable organic resistive switching device based on graphene quantum dots and polyvinylpyrrolidone composite. Org. Electron. 25, 225-231 (2015). https://doi.org/10.1016/j.orgel.2015.06.040

16. M.N. Awais, H.C. Kim, Y.H. Doh, K.H. Choi, ZrO2 flexible printed resistive (memristive) switch through electrohydrodynamic printing process. Thin Solid Films 536, 308-312 (2013). https://doi.org/10.1016/j.tsf.2013.04.003

17. A.F. Rafique, J. Haji Zaini, M.Z. Bin Esa, M.M. Nauman, Printed memory devices using electrohydrodynamic deposition technique. Appl. Phys. A 126, 134 (2020). https://doi.org/10.1007/ s00339-020-3307-0

18. M.N. Awais, N.M. Muhammad, D. Navaneethan, H.C. Kim, J. Jo, K.H. Choi, Fabrication of $\mathrm{ZrO} 2$ layer through electrohydrodynamic atomization for the printed resistive switch (memristor). Microelectron. Eng. 103, 167-172 (2013). https://doi.org/ 10.1016/j.mee.2012.09.005

19. M.N. Awais, K.H. Choi, Resistive switching mechanism in printed non-volatile $\mathrm{Ag} / \mathrm{ZrO} 2 / \mathrm{ITO}$ sandwiched structure. Electron. Lett. 51, 2147-2149 (2015). https://doi.org/10.1049/el.2014.2517

20. S. Ali, J. Bae, C.H. Lee, Printed non-volatile resistive switches based on zinc stannate (ZnSnO3). Curr. Appl. Phys. 16, 757-762 (2016). https://doi.org/10.1016/j.cap.2016.04.012

21. G.U. Siddiqui, M.M. Rehman, Y.J. Yang, K.H. Choi, A twodimensional hexagonal boron nitride/polymer nanocomposite for flexible resistive switching devices. J. Mater. Chem. C 5, 862-871 (2017). https://doi.org/10.1039/c6tc04345c

22. Y.J. Yang, M.M. Rehman, G.U. Siddiqui, K.H. Na, K.H. Choi, Effect of adding a polymer and varying device size on the resistive switching characteristics of perovskite nanocubes heterojunction. Curr. Appl. Phys. 17, 1733-1741 (2017). https://doi.org/10.1016/j. cap.2017.10.001

23. G.U. Siddiqui, M.M. Rehman, K.H. Choi, Enhanced resistive switching in all-printed, hybrid and flexible memory device based on perovskite $\mathrm{ZnSnO} 3$ via PVOH polymer. Polymer (Guildf) 100, 102-110 (2016). https://doi.org/10.1016/j.polymer.2016.07.081

24. M.M. Rehman, B.S. Yang, Y.J. Yang, K.S. Karimov, K.H. Choi, Effect of device structure on the resistive switching characteristics of organic polymers fabricated through all printed technology. Curr. Appl. Phys. 17, 533-540 (2017). https://doi.org/10.1016/j. cap.2017.01.023

25. M.M. Rehman, G.U. Siddiqui, J.Z. Gul, S.W. Kim, J.H. Lim, K.H. Choi, Resistive Switching in All-Printed, Flexible and Hybrid MoS2 -PVA Nanocomposite based Memristive Device Fabricated by Reverse Offset. Sci. Rep. 6, 36195 (2016). https://doi.org/10. 1038/srep36195

26. M.S. Onses, E. Sutanto, P.M. Ferreira, A.G. Alleyne, J.A. Rogers, Mechanisms, capabilities, and applications of high-resolution electrohydrodynamic jet printing. Small 11, 4237-4266 (2015). https://doi.org/10.1002/smll.201500593

27. B. Huber, P.B. Popp, M. Kaiser, A. Ruediger, C. Schindler, Fully inkjet printed flexible resistive memory. Appl. Phys. Lett. 110, 143503 (2017). https://doi.org/10.1063/1.4978664

28. B. Mittermeier, A. Bednar, M. Kaiser, A. Ruediger, C. Schindler, Comparison of spin-on-glass and WO 3 as an insulating layer for printed resistive memory devices. Mater. Technol. 34, 350-355 (2019). https://doi.org/10.1080/10667857.2019.1567896

29. D.H. Lien, Z.K. Kao, T.H. Huang, Y.C. Liao, S.C. Lee, J.H. He, All-printed paper memory. ACS Nano 8, 7613-7619 (2014). https://doi.org/10.1021/nn501231z

30. P. Vilmi, M. Nelo, J.V. Voutilainen, J. Palosaari, J. Pörhönen, S. Tuukkanen, H. Jantunen, J. Juuti, T. Fabritius, Fully printed memristors for a self-sustainable recorder of mechanical energy. 
Flex. Print. Electron. 1, 25002 (2016). https://doi.org/10.1088/ 2058-8585/1/2/025002

31. B. Salonikidou, T. Yasunori, B. Le Borgne, J. England, T. Shizuo, R.A. Sporea, Toward fully printed memristive elements: a-TiO2 electronic synapse from functionalized nanoparticle ink. ACS Appl. Electron. Mater. 1, 2692-2700 (2019). https://doi.org/10. 1021/acsaelm.9b00701

32. M.J. Catenacci, P.F. Flowers, C. Cao, J.B. Andrews, A.D. Franklin, B.J. Wiley, Fully printed memristors from $\mathrm{Cu}-\mathrm{SiO} 2$ core-shell nanowire composites. J. Electron. Mater. 46, 4596-4603 (2017). https://doi.org/10.1007/s11664-017-5445-5

33. X. Feng, Y. Li, L. Wang, S. Chen, Z.G. Yu, W.C. Tan, N. Macadam, G. Hu, L. Huang, L. Chen, X. Gong, D. Chi, T. Hasan, A.V.-Y. Thean, Y.-W. Zhang, K.-W. Ang, A fully printed flexible MoS2 memristive artificial synapse with femtojoule switching energy. Adv. Electron. Mater. 5, 1900740 (2019). https://doi.org/ 10.1002/aelm.201900740

34. T. Dunn, 3 - Flexographic Printing, in Flexible Packaging. ed. by T. Dunn (William Andrew Publishing, Oxford, 2015), pp. 27-37

35. H. Kipphan, Printing Technologies with Permanent Printing Master, in Handbook of Print Media: Technologies and Production Methods. ed. by H. Kipphan (Springer, Berlin Heidelberg, Berlin, Heidelberg, 2001), pp. 203-448

36. R. Abbel, Y. Galagan, P. Groen, Roll-to-roll fabrication of solution processed electronics. Adv. Eng. Mater. 20, 1701190 (2018). https://doi.org/10.1002/adem.201701190

37. G. Wang, M.A. Adil, J. Zhang, Z. Wei, Large-area organic solar cells: material requirements, modular designs, and printing methods. Adv. Mater. 31, 1805089 (2019). https://doi.org/10.1002/ adma.201805089

38. L. Gonzalez-Macia, A. Morrin, M.R. Smyth, A.J. Killard, Advanced printing and deposition methodologies for the fabrication of biosensors and biodevices. Analyst 135, 845-867 (2010). https://doi.org/10.1039/B916888E

39. Z.-L. Tseng, P.-C. Kao, M.-F. Shih, H.-H. Huang, J.-Y. Wang, S.-Y. Chu, Electrical bistability in hybrid $\mathrm{ZnO}$ nanorod/polymethylmethacrylate heterostructures. Appl. Phys. Lett. 97, 212103 (2010). https://doi.org/10.1063/1.3511756

40. C.V.V. Ramana, M.K. Moodely, V. Kannan, A. Maity, J. Jayaramudu, W. Clarke, Fabrication of stable low voltage organic bistable memory device. Sensors Actuators B Chem. 161, 684-688 (2012). https://doi.org/10.1016/j.snb.2011.11.012

41. S. Bhattacharjee, A. Roy, R. Sharma, P.K. Ahluwalia, M. Singh, Electrical characteristics of $\mathrm{ZnO}$ nanorods reinforced polymer nanocomposite thin films. AIP Conf. Proc. 1661, 80005 (2015). https://doi.org/10.1063/1.4915396

42. A.H. Jaafar, A. Gee, N.T. Kemp, Nanorods versus nanoparticles: a comparison study of $\mathrm{Au} / \mathrm{ZnO}-\mathrm{PMMA} / \mathrm{Au}$ non-volatile memory devices showing the importance of nanostructure geometry on conduction mechanisms and switching properties. IEEE Trans. Nanotechnol. 19, 236-246 (2020). https://doi.org/10.1109/ TNANO.2019.2949759

43. S.P. Singh, S.K. Sharma, D.Y. Kim, Carrier mechanism of $\mathrm{ZnO}$ nanoparticles-embedded PMMA nanocomposite organic bistable memory device. Solid State Sci. 99, 106046 (2020). https://doi. org/10.1016/j.solidstatesciences.2019.106046

44. J.-W. Lee, W.-J. Cho, Fabrication of resistive switching memory based on solution processed PMMA $\}$ - HfOxblended thin films. Semicond. Sci. Technol. 32, 25009 (2017). https://doi.org/10. 1088/1361-6641/aa5133

45. I.J. Baek, W.J. Cho, Resistive switching characteristics of solution-processed organic-inorganic blended films for flexible memory applications. Solid State Electron. 140, 129-133 (2018). https://doi.org/10.1016/j.sse.2017.10.030

46. P.K. Sarkar, S. Bhattacharjee, M. Prajapat, A. Roy, Incorporation of $\mathrm{SnO} 2$ nanoparticles in PMMA for performance enhancement of a transparent organic resistive memory device. RSC Adv. 5, 105661-105667 (2015). https://doi.org/10.1039/C5RA15581A

47. M.A.U. Khalid, S.W. Kim, J. Lee, A.M. Soomro, M.M. Rehman, B.-G. Lee, K.H. Choi, Resistive switching device based on SrTiO3/PVA hybrid composite thin film as active layer. Polymer (Guildf) 189, 122183 (2020). https://doi.org/10.1016/j.polymer. 2020.122183

48. W.T. Kim, J.H. Jung, T.W. Kim, D.I. Son, Current bistability and carrier transport mechanisms of organic bistable devices based on hybrid Ag nanoparticle-polymethyl methacrylate polymer nanocomposites. Appl. Phys. Lett. 96, 253301 (2010). https://doi.org/ 10.1063/1.3453661

49. K. Rajan, I. Roppolo, K. Bejtka, A. Chiappone, S. Bocchini, D. Perrone, C.F. Pirri, C. Ricciardi, A. Chiolerio, Performance comparison of hybrid resistive switching devices based on solution-processable nanocomposites. Appl. Surf. Sci. 443, 475-483 (2018). https://doi.org/10.1016/j.apsusc.2018.03.008

50. M. Kim, H. Jung, Y.-H. Kim, C.-J. Kang, T.-S. Yoon, H.H. Lee, Resistive switching of in situ polymerized polystyrene matrix copolymerized with alkanedienyl passivated Si nanoparticles. Microelectron. Eng. 136, 26-30 (2015). https://doi.org/10.1016/j. mee.2015.03.043

51. J. Mangalam, S. Agarwal, A.N. Resmi, M. Sundararajan, K.B. Jinesh, Resistive switching in polymethyl methacrylate thin films. Org. Electron. 29, 33-38 (2016). https://doi.org/10.1016/j.orgel. 2015.11.017

52. C. Schwarz, M. Kaiser, S.F. Jacob, C. Schindler, Partially printed resistive memory cells on rigid and flexible substrates. Phys. Status Solidi Appl. Mater. Sci. 213, 1353-1357 (2016). https://doi. org/10.1002/pssa.201532851

53. G.C. Schmidt, P.M. Panicker, X. Qiu, A.J. Benjamin, R.A. Quintana Soler, I. Wils, A.C. Hübler, Paper-embedded roll-to-roll mass printed piezoelectric transducers. Adv. Mater. 33, 2006437 (2021). https://doi.org/10.1002/adma.202006437

54. S.G. Adoor, L.S. Manjeshwar, K.S.V.K. Rao, B.V.K. Naidu, T.M. Aminabhavi, Solution and solid-state blend compatibility of poly(vinyl alcohol) and poly(methyl methacrylate). J. Appl. Polym. Sci. 100, 2415-2421 (2006). https://doi.org/10.1002/app. 23337

55. M. Hösel, F.C. Krebs, Large-scale roll-to-roll photonic sintering of flexo printed silver nanoparticle electrodes. J. Mater. Chem. 22, 15683-15688 (2012). https://doi.org/10.1039/C2JM32977H

56. M.L. Morgan, A. Holder, D.J. Curtis, D. Deganello, Formulation, characterisation and flexographic printing of novel Boger fluids to assess the effects of ink elasticity on print uniformity. Rheol. Acta 57, 105-112 (2018). https://doi.org/10.1007/s00397-017-1061-9

57. P.G. Saffman, G.I. Taylor, The penetration of a fluid into a porous medium or Hele-Shaw cell containing a more viscous liquid. Proc. R Soc. London Ser. A Math. Phys. Sci. 245, 312-329 (1958). https://doi.org/10.1098/rspa.1958.0085

58. S. Walheim, M. Böltau, J. Mlynek, G. Krausch, U. Steiner, Structure formation via polymer demixing in spin-cast films. Macromolecules 30, 4995-5003 (1997). https://doi.org/10.1021/ma961 9288

59. K. Krishnan, M. Aono, T. Tsuruoka, Kinetic factors determining conducting filament formation in solid polymer electrolyte based planar devices. Nanoscale 8, 13976-13984 (2016). https://doi.org/ $10.1039 / \mathrm{c} 6 \mathrm{nr} 00569 \mathrm{a}$

60. S. Gao, C. Song, C. Chen, F. Zeng, F. Pan, Dynamic processes of resistive switching in metallic filament-based organic memory devices. J. Phys. Chem. C 116, 17955-17959 (2012). https://doi. org/10.1021/jp305482c

61. Z. Wang, F. Zeng, J. Yang, C. Chen, F. Pan, Resistive switching induced by metallic filaments formation through Poly(3,4-ethylene-dioxythiophene):Poly(styrenesulfonate). ACS Appl. Mater. Interfaces 4, 447-453 (2012). https://doi.org/10.1021/am201518v 
62. Y.-C. Lai, D.-Y. Wang, I.-S. Huang, Y.-T. Chen, Y.-H. Hsu, T.-Y. Lin, H.-F. Meng, T.-C. Chang, Y.-J. Yang, C.-C. Chen, F.-C. Hsu, Y.-F. Chen, Low operation voltage macromolecular composite memory assisted by graphene nanoflakes. J. Mater. Chem. C 1, 552-559 (2013). https://doi.org/10.1039/C2TC00010E

63. I. Valov, R. Waser, J.R. Jameson, M.N. Kozicki, Electrochemical metallization memories - Fundamentals, applications, prospects. Nanotechnology 22, 254003 (2011)

64. S.-H. Lee, H.-L. Park, C.-M. Keum, I.-H. Lee, M.-H. Kim, S.-D. Lee, Organic flexible memristor with reduced operating voltage and high stability by interfacial control of conductive filament growth. Phys. Status Solidi Rapid Res. Lett. 13, 1900044 (2019). https://doi.org/10.1002/pssr.201900044

65. Y. Qi, Z. Shen, C. Zhao, C.Z. Zhao, Effect of electrode area on resistive switching behavior in translucent solution-processed
AlOx based memory device. J. Alloys Compd. 822, 153603 (2020). https://doi.org/10.1016/j.jallcom.2019.153603

66. M.T. Dang, J. Lefebvre, J.D. Wuest, Recycling indium tin oxide (ITO) electrodes used in thin-film devices with adjacent holetransport layers of metal oxides. ACS Sustain. Chem. Eng. 3, 3373-3381 (2015). https://doi.org/10.1021/acssuschemeng.5b010 80

Publisher's Note Springer Nature remains neutral with regard to jurisdictional claims in published maps and institutional affiliations. 